\title{
Predicate-based Filtering of XPath Expressions
}

\author{
Shuang Hou \\ H.-Arno Jacobsen \\ University of Toronto, Canada $\{$ shou@cs, jacobsen@eecg $\}$.utoronto.ca
}

\begin{abstract}
The XML/XPath filtering problem has found wide-spread interest. In this paper, we propose a novel algorithm for solving it. Our approach encodes XPath expressions (XPEs) as ordered sets of predicates and translates XML documents into sets of tuples, which are evaluated over these predicates. Predicates representing overlapping portions of XPEs are stored and processed once, thus fully exploiting potential overlap in XPEs. We experimentally evaluate the performance of our algorithm, demonstrating its scalability to millions of XPEs, with matching performance in the millisecond range. We show interesting trade-offs to alternative approaches.
\end{abstract}

\section{Introduction}

XML has been widely advocated as data representation format of choice for selective information dissemination applications and for content-based message routing $[7,5,10,4]$. In these scenarios, it is expected that the filtering engines have to be capable of processing several millions of filter expressions over high XML document input rates.

For selective information dissemination applications, an XPath filter expression designates an entity's (user or system) interest to receive information of the specified nature. The XML document constitutes the content to be selectively disseminated. XPath expressions ${ }^{1}$ have been widely proposed for modeling such filters $[2,7,5,10,9,4,13]$. An XPath expression (XPE) can specify filter constraints on the document's structure, its attributes, and its content. Filter selectivity on a very fine-grained level is thus possible.

The XML/XPath filtering problem determines the set of matching XPEs among all expressions to be processed for a given input XML document. It is this problem that we set out to solve with a novel filtering algorithm. The algorithm we propose is fundamentally different from existing approaches $[2,7,5,10,9,4,13]$, which are based on finite state machines, nodeterministic finite state machines, pushdown automaton, trie-based indices, or relational database

${ }^{1}$ http://www.w3.org/TR/xpath20 querying techniques. The major challenge of this filtering problem is to efficiently handle a large number of XPEs. Our goal and main difference to related approaches is to exploit the overlap among XPEs as much as possible. Our techniques go beyond exclusively considering prefix overlap. The main idea of our algorithm is to translate XPEs into ordered sets of predicates, such that the common parts among XPEs will be stored and processed only once, and the partial matching result will be shared among all expressions that contain these common parts. For example, in the two XPEs $s_{1}: a / b / c / d$ and $s_{2}: b / / b / c$, we translate the overlapping parts, $b / c$, into one predicate. This predicate will be evaluated against the XML document only once. It is also shared by other expressions that contain it.

The first contribution of this paper is a completely novel algorithm for solving the XML filtering problem. In a second contribution, we propose several refinements and optimizations for our approach, such as the predicate index, the prefix covering index, the access predicate scheme, and extensions for processing nested path and attribute-based filters. Third, we perform a thorough experimental analysis that evaluates the performance of our algorithm under varying experimental conditions. We demonstrate the scalability of the algorithm to millions of XPEs, and present a detailed trade-off analysis against two alternative techniques from different categories of the solution space of the filtering problem.

\section{Related Work}

Related approaches can be broadly classified into automaton-based approaches and index-based approaches. Automaton-based approaches $[2,7,9,10]$ build an automaton based on the XPEs processed by the system. The transitions in the automaton are triggered by the tags of the XML document path being processed. XFilter [2] treats each XPE as a finite state machine. This approach is not able to adequately handle overlap, especially, prefix overlap between expressions. YFilter [7] extends XFilter by building a non-deterministic finite automaton (NFA) for all XPEs in the system. It honors common prefixes among expressions. Each final state of the NFA is associated with a list of expressions. The parsing of the XML document, one tag 
at a time, triggers the transitions in the NFA. Whenever a final state is reached, it means that the corresponding expressions are matched by the XML document processed. The difference between a traditional NFA and the YFilter data structure is that the execution of the YFilter NFA does not stop when the first final state is reached, but continues until all possible accepting states have been visited. Thus, the algorithm can determine all matching expressions stored in the NFA. The YFilter [7] paper demonstrates superior performance to XTrie [5], which is the reason we chose YFilter for our performance comparisons. Another automatonbased approach, XPush [10], lazily constructs a single deterministic pushdown automaton for XPEs in the system. A drawback of the approach for online selective information processing is the difficulty in adding or deleting queries from the automaton constructed.

The index-based algorithms $[4,5,12]$ take advantage of precomputed schemes on either the XML documents or the XPath expressions. XTrie [5] proposes a trie-based index structure, which decomposes the XPEs to substrings that only contain parent-child operators. As a result, the processing of these common substrings among queries can be shared. Despite this sharing, YFilter [7] has been demonstrated to have better performance on certain workloads. Lakshman et al. [12] propose an index structure that manages XPEs for solving the filtering problem. The algorithms requires two passes over an XML document processed. Index-Filter [4] addresses the problem of obtaining all matches for each expression stored in the system. It answers multiple XML path queries by building indexes over the XML document elements either in a subcomputation stage or on the fly, which avoids processing large portions of the input document that are guaranteed not to be part of any match. The algorithm takes advantage of a prefix tree representation of the set of XML queries to share the computation during multiple evaluations. It is efficient especially in the case of large XML documents or set of documents.

\section{XPath and XML Encoding}

In this section, we motivate and describe the encoding of XPEs with a predicate calculus and the encoding of XML documents as sets of tuples. Our algorithm, described in the following section, efficiently evaluates the tuples over the predicates to determine the matching XPEs.

\subsection{Basic Idea}

The basic idea underlying our encoding is to record the position information of each tag and the relative position information for each two adjacent tags in the XPEs and XML documents, respectively. From this we can determine whether the position information recorded for each XPE is matched by the position information represented in the current XML document.

Each XPE is translated into an ordered set of predicates, where each predicate is an (attribute, operator, value)-triple that expresses a constraint over the value of the attribute (i.e., the position of the tag). More formally, an XPE is described as $s:\left(a_{1}, o_{1}, v_{1}\right) \mapsto\left(a_{2}, o_{2}, v_{2}\right) \mapsto \ldots \mapsto$ $\left(a_{n}, o_{n}, v_{n}\right)$, where $a_{i}$ represents either a tag name variable or a pair of tag name variables, $o_{i}$ represents a relational operator and “ $\mapsto$ " represents the sequence of predicates, that is, $\left(a_{i-1}, o_{i-1}, v_{i-1}\right)$ should be ahead of $\left(a_{i}, o_{i}, v_{i}\right)$. For example, $a / * / b / / c$ is translated to $\left(d\left(p_{a}, p_{b}\right),=, 2\right) \mapsto$ $\left(d\left(p_{b}, p_{c}\right), \geq, 1\right)$, where each predicate encodes the relative position information of each two adjacent tags (the predicates will be discussed shortly.) The order-preserving requirement imposed by the sequencing of predicates is an essential part of our approach and will be clarified in the sequel.

In our approach, we use the common interpretation of an XML document as a tree of nodes and consider each path from the root node in this tree to a leaf node, separately. ${ }^{2}$ These paths are "collected" in the parsing stage. In our implementation we use a SAX parser and extract one path at a time from the document. This path extraction adds a negligible amount of overhead, as we illustrate in our experiments. Thus, we decompose each XML document into a set of XML paths and each path is encoded as a set of (attributevalue)-pairs. where each pair is, either a single XML tag name, $a_{i}$, and its position value, $v_{i}$, in the document path, or a special attribute length representing the length of this XML path. For example, the XML path, $e=\left(t_{1}, t_{2}, \ldots, t_{n}\right)$, in our encoding is $\left\{(\right.$ length,$\left.n),\left(t_{1}, 1\right),\left(t_{2}, 2\right), \ldots,\left(t_{n}, n\right)\right\}$. Further examples will follow in Section 3.3.

For each XML document, we evaluate all its XML paths against all XPEs in the system. An XPE is matched by a given XML document iff ${ }^{3}$ the evaluation of the XPE over the XML document is a non-empty set of nodes in the XML tree. Our data structure and algorithm exploit overlap in XPEs by storing and evaluating unique predicates only.

If any XPE, $s_{i}$, in the system is matched by any document path of an incoming XML document, $D=$ $\left\{e_{1}, e_{2}, \ldots, e_{n}\right\}$, we say the XPE is matched by this document. The objective of this matching semantic is to filter out XML documents matching any XPE stored in the system. Note that the XPEs, $s_{i}$, are single-path expressions in the following discussion. The matching of nested path expressions is discussed in [11].

\footnotetext{
${ }^{2}$ For processing nested path filters, we need to keep state between processing different paths. The interested reader is referred to the extended version of this paper [11]

${ }^{3}$ We will use the notation iff to mean "if and only if" in this paper.
} 


\subsection{XPath Expression Encoding}

First, we present our predicate language for encoding XPE operators. In this section, we focus on parent-child operator $(/)$, wildcard operator $(*)$, and ancestor-descendant operator $(/ /)$. We then show how to represent entire XPEs in this language. We will discuss how to support attributebased and nested path filters with our predicate language in [11]. This extensibility demonstrates the principle for adding other XPath language support to our approach.

Predicate Language: We use an extensible predicate language to express constraints over the absolute and the relative position of tags in the XPEs. We define the following four types of predicates to represent absolute XPEs, relative XPEs, wildcards in XPEs, and //-operators. Later, we show how to extend this language to process attribute filters in XPEs and nested path filters.

Absolute predicates: The predicate, $\left(p_{t}, o p, v\right)$, represents a constraint on the position of the tag, $t$, in the XPE. The operator, $o p$, can be either $=$ or $\geq$. For the equality operator, this predicate represents the absolute position of a tag in the absolute XPE (i.e., an XPE explicitly starting at the root element and not including descendant operators). For example, in the XPE, $/ * / t_{1}$, the predicate, $\left(p_{t_{1}},=, 2\right)$, is used to represent the absolute position information of tag $t_{1}$ in $s$. For the greater-than-or-equal operator, this predicate represents the tag, which appears after some descendant operator in the absolute XPE, or represents the first tag in the relative XPE, which is not a wildcard. For example, in the absolute XPE, $/ * / / t_{1},\left(p_{t_{1}}, \geq, 2\right)$ is used to represent the position information of tag $t_{1}$. In the relative XPE, $* / * / t_{1}$, the predicate, $\left(p_{t_{1}}, \geq, 3\right)$ is used to represent the position information of tag $t_{1}$.

Relative predicates: The predicate, $\left(d\left(p_{t_{1}}, p_{t_{2}}\right), o p, v\right)$, represents a constraint between the relative position of the $\operatorname{tag}, t_{2}$, with respect to the tag, $t_{1}$. The operator op can be either $=$ or $\geq$. For the equality operator, this predicate represents tags in XPEs that are not linked by descendant operators. For example, for $t_{1} / * / t_{2}$, the predicate, $\left(d\left(p_{t_{1}}, p_{t_{2}}\right),=, 2\right)$ is used to represent the relative position information between the tag $t_{1}$ and $t_{2}$. Note that the order between these tags in the path is important. The predicate $\left(d\left(p_{t_{1}}, p_{t_{2}}\right),=, 2\right)$ indicates that the tag $t_{1}$ should appear two location steps before the tag $t_{2}$ in the XML path. For the greater-than-or-equal operator, this predicate represents tags that are linked by descendant operators. For example, in the XPE $t_{1} / / t_{2}$, the predicate, $\left(d\left(p_{t_{1}}, p_{t_{2}}\right), \geq, 1\right)$ expresses that the tag, $t_{1}$, must appear more than one location step before the tag, $t_{2}$, in any matching document path.

End-of-path predicates: The predicate, $\left(p_{t}^{-1}, \geq, v\right)$, represents a constraint on the position of the tag, $t$, relative to the end of the XML path. It is for those tags in the absolute or relative XPE, which are followed by wildcards only. For example, for $/ t_{1} / * / *$, not only do we need the predicate, $\left(p_{t_{1}},=, 1\right)$, to represent the position constraint of tag, $t_{1}$, we also need the predicate, $\left(p_{t_{1}}^{\dashv}, \geq, 2\right)$, to represent the position constraint of this tag relative to the end of the XML path. There must be two or more tags following $t_{1}$ in a matching XML path to satisfy the expression.

Length-of-expression predicates: The predicate, (length, $\geq, v)$, is a special predicate, which does not refer to any tag names and their positions, but to the length of the XPE. It represents a constraint on the length of the XML path. It is for those XPEs that contain only wildcards. For example, the XPE, $s_{1}: / * / * / *$, is translated into (length, $\geq, 3$ ), which indicates that all XML paths containing enough tags will match this XPE. Note that the expression $s_{2}: * / * / *$ has the same mapping, since both of them actually require that the length of the XML path is at least 3 . We do not distinguish these two kinds of expressions in this paper, since no distinction is required to satisfy the matching semantic we set out to solve (i.e., any document path of length at least 3 will correctly match both expressions.)

Mapping XPEs to Predicates: This section illustrates, first, through various examples and then generically how different XPEs are translated to the ordered sets of predicates of the types defined in the previous section. These predicates record the position information of the first nonwildcarded location step and the relative position information between every two adjacent tags. The objective is to record just enough information to uniquely represent each XPE. The mapping also honors overlap between different XPEs and represents overlapping parts of expressions with the same predicates. Our algorithm only stores and processes unique predicates once, thus leveraging the overlap in expressions.

Simple XPEs: A simple XPE only contains parent-child operators. It is represented with absolute and relative predicates as illustrated below.

\begin{tabular}{|l|l|}
\hline XPE & Ordered predicates \\
\hline$s_{1}: / a / b$ & $\left(p_{a},=, 1\right) \mapsto\left(d\left(p_{a}, p_{b}\right),=, 1\right)$ \\
\hline$s_{2}: a$ & $\left(p_{a}, \geq, 1\right)$ \\
\hline$s_{3}: a / a / b$ & $\left(d\left(p_{a}, p_{a}\right),=, 1\right) \mapsto\left(d\left(p_{a}, p_{b}\right),=, 1\right)$ \\
\hline
\end{tabular}

For $s_{3}$, we do not need the predicate $\left(p_{a}, \geq, 1\right)$ for the first tag, $a$, since other predicates already indicate that the constraint on the position of the first tag should be at least 1 . Note, for certain XPEs alternative encodings come to mind. However, it is these encodings here for which we can prove the correctness of encodings [11] (due to space limitations the proof is available from our web site.)

The general absolute XPE, $/ t_{1} / t_{2} / \ldots / t_{n-1} / t_{n}$, is encoded as $\left(p_{t_{1}},=, 1\right) \mapsto\left(d\left(p_{t_{1}}, p_{t_{2}}\right),=, 1\right) \mapsto \ldots \mapsto(d($ $\left.\left.p_{t_{n-1}}, p_{t_{n}}\right),=, 1\right)$. The general relative XPE, $t_{1} / t_{2} / \ldots /$ $t_{n-1} / t_{n}$, is encoded as $\left(d\left(p_{t_{1}}, p_{t_{2}}\right),=, 1\right) \mapsto \ldots \mapsto(d($ $\left.\left.p_{t_{n-1}}, p_{t_{n}}\right),=, 1\right)$.

Wildcards in XPEs: Wildcards can appear at the begin- 
ning, the middle and the end of an XPE. In our encoding of XPEs with wildcards, we simply bypass the wildcarded location steps as illustrated below.

\begin{tabular}{|l|l|}
\hline XPE & Ordered predicates \\
\hline$s_{4}: / a / * / * / b$ & $\left(p_{a},=, 1\right) \mapsto\left(d\left(p_{a}, p_{b}\right),=, 3\right)$ \\
\hline$s_{5}: / * / a / b$ & $\left(p_{a},=, 2\right) \mapsto\left(d\left(p_{a}, p_{b}\right),=, 1\right)$ \\
\hline$s_{6}: / * / * / * / *$ & $($ length,$\geq, 4)$ \\
\hline$s_{7}: a / b / * / *$ & $\left(d\left(p_{a}, p_{b}\right),=, 1\right) \mapsto\left(p_{b}^{-}, \geq, 2\right)$ \\
\hline$s_{8}: * / * / a / * / b$ & $\left(p_{a}, \geq, 3\right) \mapsto\left(d\left(p_{a}, p_{b}\right),=, 2\right)$ \\
\hline$s_{9}: * / * / * / *$ & $($ length,$\geq, 4)$ \\
\hline
\end{tabular}

In the expression, $s_{8}$, we need the predicate $\left(p_{a}, \geq, 3\right)$ to represent the position constraint of tag, $a$, since the other predicates do not represent any position information for the first non-wildcarded location step.

The general absolute XPE, / * /... $/ * / t_{1} / \ldots / t_{i} / *$ $/ \ldots / * / t_{i+1} / \ldots / t_{n} / * / \ldots / *$, is encoded as $\left(p_{t_{1}},=, m_{1}\right) \mapsto$ $\left(d\left(p_{t_{1}}, p_{t_{2}}\right),=, 1\right) \mapsto \ldots \mapsto\left(d\left(p_{t_{i-1}}, p_{t_{i}}\right),=, 1\right) \mapsto$ $\left(d\left(p_{t_{i}}, p_{t_{i+1}}\right),=, m_{2}\right) \mapsto \ldots \mapsto\left(d\left(p_{t_{n-1}}, p_{t_{n}}\right),=, 1\right) \mapsto$ $\left(p_{t_{n}}^{\dashv}, \geq, m_{3}\right)$, where $m_{1}, m_{2}$ and $m_{3}$ are consecutive wildcards appearing at the beginning, middle and end of the $\mathrm{XPE}$, respectively. The mapping for a general relative XPE with wildcards is similar, except that the first predicate is replaced by the predicate, $\left(p_{t_{1}}, \geq, m_{1}\right)$.

Descendant operator in XPEs: Descendant operators indicate that there are more than one location step between two tags as illustrated below.

\begin{tabular}{|l|l|}
\hline XPE & Ordered predicates \\
\hline$s_{10}: / a / / b$ & $\left(p_{a},=, 1\right) \mapsto\left(d\left(p_{a}, p_{b}\right), \geq, 1\right)$ \\
\hline$s_{11}: / * / b / / c$ & $\left(p_{b},=, 2\right) \mapsto\left(d\left(p_{b}, p_{c}\right), \geq, 1\right)$ \\
\hline$s_{12}: a / b / / c$ & $\left(d\left(p_{a}, p_{b}\right),=, 1\right) \mapsto\left(d\left(p_{b}, p_{c}\right), \geq, 1\right)$ \\
\hline$s_{13}: * / a / * /$ & $\left(p_{a}, \geq, 2\right) \mapsto\left(d\left(p_{a}, p_{b}\right),=, 2\right) \mapsto\left(d\left(p_{b}\right.\right.$, \\
$b / / c / * / *$ & $\left.\left.p_{c}\right), \geq, 1\right) \mapsto\left(p_{c}^{-}, \geq, 2\right)$ \\
\hline
\end{tabular}

The general form of an absolute XPE with descendant operator(s), $/ t_{1} / \ldots / t_{i} / / t_{i+1} / \ldots / t_{n}$, is encoded as $\left(p_{t_{1}},=\right.$ $, 1) \mapsto\left(d\left(p_{t_{1}}, p_{t_{2}}\right),=, 1\right) \mapsto \ldots \mapsto\left(d\left(p_{t_{i-1}}, p_{t_{i}}\right),=, 1\right) \mapsto$ $\left(d\left(p_{t_{i}}, p_{t_{i+1}}\right), \geq, 1\right) \mapsto \ldots \mapsto\left(d\left(p_{t_{n-1}}, p_{t_{n}}\right),=, 1\right)$, where the descendant operator can appear at any location step of the XPE one or more than one time. The encoding for the general relative $X P E$ with descendant operators is the same, except for missing first predicate.

As a result, the common part among the XPEs are mapped to the same one or several predicates, which will be stored and evaluated only once during the matching. For example, $a / b$ is translated into only one predicate $\left(d\left(p_{a}, p_{b}\right),=, 1\right)$ in the above examples in spite of the position it appears in the XPEs.

It is important to note that our mapping is not predicateorder invariant. That is, the order of predicates can not be changed because different tags may have the same name, and “ $\mapsto$ " determines the sequence of predicates appearing in the mapping, which is the reason for the use of an ordered set, rather than a traditional set in our formaliza- tion. For example, $\left(d\left(p_{a}, p_{c}\right),=, 1\right) \mapsto\left(d\left(p_{c}, p_{a}\right),=, 2\right)$ $\mapsto\left(d\left(p_{a}, p_{c}\right), \geq, 1\right)$ is used to represent the XPE, $s_{1}$ : $a / c / * / a / / c$, where the first tag, $a$, and the third tag, $a$, are different tags but have the same name. We identify them by looking at $\left(d\left(p_{a}, p_{c}\right),=, 1\right)$ and $\left(d\left(p_{a}, p_{c}\right), \geq, 1\right)$, which indicate that the first tag, $a$, is followed by tag, $/ c$, and another tag, $a$, is followed by $/ / c$. If we change the order of these two predicates, say $\left(d\left(p_{a}, p_{c}\right), \geq, 1\right) \mapsto\left(d\left(p_{c}, p_{a}\right),=\right.$ $, 2) \mapsto\left(d\left(p_{a}, p_{c}\right),=, 1\right)$, another XPE, $s_{2}: a / / c / * / a / c$, is represented. That is, we use the order of these predicates to represent the sequence of tags appearing in the expression.

\subsection{XML Document Encoding}

An XML document, $D$, consists of a number of document paths, $D=\left(e_{1}, e_{2}, \ldots, e_{n}\right)$. Each document path, $e_{i}=\left(t_{1}, t_{2}, \ldots, t_{n}\right)$, consists of a sequence of tag names, $t_{i}$, optional attributes and corresponding values, and the content associated with each tag. For simplicity we only refer to the tag names in the above formalization.

The intuition behind our document path encoding is that we translate each XML document path to a set of (attribute, value)-pairs, which are evaluated against the XPE predicates. Our encoding records the information of the path length and the position of each tag in the document path. We translate each XML document path, $e=\left(t_{1}, t_{2}, \ldots, t_{n}\right)$, to the following set of tuples: (length, $n),\left(t_{1}, 1\right),\left(t_{2}, 2\right), \ldots,\left(t_{n}, n\right)$, where (length, $\left.n\right)$ represents the length of the XML path, which is required to evaluate the predicates $\left(p_{t}^{-1}, \geq, v\right)$ and (length, $\left.\geq, v\right)$. The pair $\left(t_{i}, i\right)$ determines that the position of tag $t_{i}$ is $i$ in this path. This is required to evaluate the predicates $\left(p_{t_{i}}, o p, v\right)$ and $\left(p_{t_{i}}^{\dashv}, \geq, v\right)$. In order to evaluate the predicate $\left(d\left(p_{t_{i}}, p_{t_{j}}\right), o p, v\right)$, two pairs, $\left(t_{i}, i\right)$ and $\left(t_{j}, j\right)$, are required at the same time to determine the distance between $\operatorname{tag} t_{i}$ and $t_{j}$. The interpretation as (attribute, value)-pairs derives from the fact that "length" and the tag name are attribute designators. The range of the value is $[1, n]$ ( $n$ is the length of the XML path). We refer to this set of (attribute, value)-pairs for each document path as publication. Based on this encoding, all predicates are evaluated against each tuple or a pair of tuples in the publication according to a set of predefined rules for predicate matching.

In our encoding, we distinguish the case of different tags with the same name occurring in the same XML document path. Each tag is annotated with an occurrence number, for that purpose, which counts the number of times this tag was already present in this path.

Example 1: Consider the path, $e=(a, b, c, a, b, c)$, which is annotated with superscripted occurrence numbers, $e=\left(a^{1}, b^{1}, c^{1}, a^{2}, b^{2}, c^{2}\right)$. It is translated into the following publication: (length, 6$),\left(a^{1}, 1\right),\left(b^{1}, 2\right),\left(c^{1}, 3\right)$, $\left(a^{2}, 4\right),\left(b^{2}, 5\right),\left(c^{2}, 6\right)$, where $\left(a^{2}, 4\right)$ indicates that the second occurring $a$ in this document path is at position 4 . 
This encoding is built in the XML document parsing stage and does not require additional processing, except for collecting the occurrence numbers for each tag appearing in the current path. In our implementation this is based on a hashtable that keeps track of the already for the current path seen tag names.

\section{Matching Algorithm}

In this section, we discuss the matching algorithm. It has two stages. First, the predicate matching stage determines all matching predicates and, second, the XPath expression matching stage. The latter stage computes the matching XPEs based on the matching predicate information determined in the first stage. We discuss both stages in turn.

\subsection{Predicate Matching}

\subsubsection{Predicate Evaluation}

Each of the four predicates is evaluated over a document path according to the following rules.

Absolute predicates: The predicate, $\left(p_{t}, o p, v\right)$, is matched by a given tuple iff the tuple is $\left(t, v^{\prime}\right)$, and it satisfies the relation $v^{\prime}$ op $v$.

Relative predicates: The predicate, $\left(d\left(p_{t_{1}}, p_{t_{2}}\right), o p, v\right)$, is matched by a given pair of tuples iff the tuples are $\left(t_{1}, v_{1}\right)$, $\left(t_{2}, v_{2}\right)$, and they satisfy the relation $\left(v_{2}-v_{1}\right)$ op $v$. For example, given tuples $(a, 2)$ and $(b, 6),\left(d\left(p_{a}, p_{b}\right),=, 2\right)$ is not matched since $6-2=2$ does not hold.

End-of-path predicates: The predicate, $\left(p_{t}^{-1}, \geq, v\right)$, is matched by a given tuple iff the tuple is $\left(t, v^{\prime}\right)$, and it satisfies the relation $l-v^{\prime} \geq v$ ( $l$ is the length value).

Length-of-expression predicates: The predicate, (length, $\geq, v$ ), is matched by a given tuple iff the tuple is (length, $v^{\prime}$ ), and it satisfies the relation $v^{\prime} \geq v$.

For each tuple in a publication (i.e., document path), we record the matching predicates and the occurrence number of the tag associated with each match. Both are used to determine the matching XPEs in the second stage of the algorithm.

Table 1. Predicate Matching Result

\begin{tabular}{|c|c|c|}
\hline XPE & Predicates & Matching Results \\
\hline \multirow{2}{*}{$a / / b / c$} & $\left(d\left(p_{a}, p_{b}\right), \geq, 1\right)$ & $\left(\mathbf{a}^{1}, \mathbf{b}^{1}\right),\left(a^{1}, b^{2}\right),\left(a^{2}, b^{2}\right)$ \\
\cline { 2 - 3 } & $\left(d\left(p_{b}, p_{c}\right),=, 1\right)$ & $\left(\mathbf{b}^{\mathbf{1}}, \mathbf{c}^{\mathbf{1}}\right),\left(b^{2}, c^{2}\right)$ \\
\hline \multirow{2}{*}{$c / / b / / a$} & $\left(d\left(p_{c}, p_{b}\right), \geq, 1\right)$ & $\left(c^{1}, b^{2}\right)$ \\
\cline { 2 - 3 } & $\left(d\left(p_{b}, p_{a}\right), \geq, 1\right)$ & $\left(b^{1}, a^{2}\right)$ \\
\hline
\end{tabular}

Example 2: Considering the XML path from Example 1 and the two XPEs: $a / / b / c$ and $c / / b / / a$, the individual predicate matching results are shown in Table 1 . For expression $a / / b / c$, which translates to $\left(d\left(p_{a}, p_{b}\right), \geq\right.$ $, 1) \mapsto\left(d\left(p_{b}, p_{c}\right),=, 1\right)$, each predicate with its corresponding matching results is shown. The combination of two boldface matching results is one of the true matches for $a / / b / c$. From these results, it can be seen that not all combinations of the predicate matching results constitutes a true XPE match. For example, predicate matching results $\left(a^{1}, b^{2}\right)$ and $\left(b^{1}, c^{1}\right)$ are not a true XPE match, since the occurrence numbers (i.e., superscripts) indicate that the first predicate is matched by the second $b$ in the XML path, while the second predicate is matched by the first $b$ in the path. The same situation occurs in the other expression, which indicates that there is no match for $c / / b / / a$. The occurrence determination algorithm, described in Section 4.2.1, will determine whether at least one true match exists, among the individual predicate matching results, or not for each XPE.

\subsubsection{Predicate Index}

We maintain a predicate index that manages distinct predicates through multiple stages of hashtables. Suppose that we have two XPEs, $/ a / * / c$ and $* / a / * / c / * / * / *$, the common part $a / * / c$ is represented as $\left(d\left(p_{a}, p_{c}\right),=, 2\right)$ and only stored once in our predicate index, as shown in Figure 1. All predicates are managed in the first stage of the index according to their type. For absolute predicates, i.e., $\left(p_{t}, o p, v\right)$, we form hash keys by using the tag name to access a separate array per operator that stores predicate identifiers (pids). Each array is indexed by the predicate's value. The length of the array depends on the maximum length of the XPEs supported by the system. For relative predicates, i.e., $\left(d\left(p_{t_{1}}, p_{t_{2}}\right), o p, v\right)$, we form a first-level hash key with the first tag and a second-level hash key with the second tag. The first-level hashtable indexes into a second-level table, which indexes into the same structure as the table for managing absolute predicates. For end-of-path predicates, i.e., $\left(p_{t}^{-1}, \geq, v\right)$, we form a hash key based on the tag and manage one array per tag that keeps track of all predicates of this type. For length-of-path predicates, i.e., (length, $\geq, v$ ), we only keep an array and do not need additional keys since length and $\geq$ are fixed.

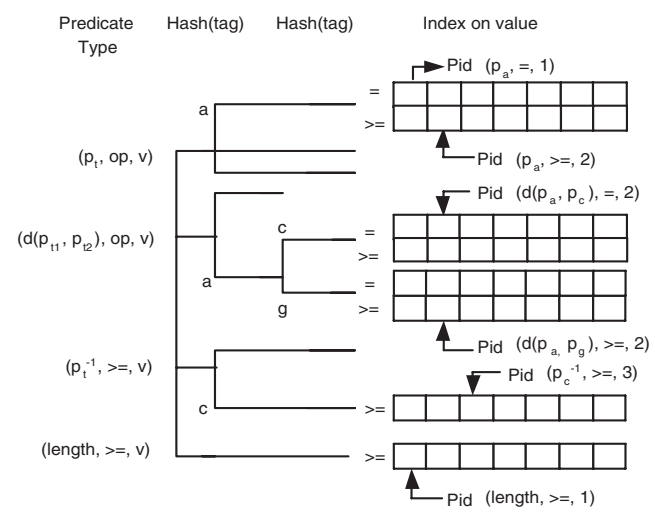

Figure 1. Predicate Index

A predicate is inserted into the index by hashing on tag name(s) and indexing into the corresponding array with the 
predicate value. If a pid is found, it is returned, indicating that the exact same predicate is already stored in the system, otherwise a new pid is created for this predicate and stored at the indexed location in the array.

Based on predicate matching rules defined in Section 4.1.1, the predicate matching is straightforward by using the predicate index. For absolute predicates, given each tuple in the document path, a matching predicate is identified by checking for existing pids in the associated array at the index position identified by the value in this tuple (for equality predicates) and at all index positions in the array larger than this value (for greater-than-or-equal predicates). For end-of-path and length-of-expression predicates, the evaluation is similar except that the length of document path is applied. For relative predicates, the evaluation must correlate a pair of tuples and the index position is identified by the difference of the positions of the second-level and first-level tags in the document path.

\subsection{XPath Expression Matching}

The previous predicate matching stage results in a set of matched predicates. For each predicate we record matching occurrences, i.e., an occurrence number of the tag that matches the predicate. From this information we compute the matching XPEs. In this section, we first present the algorithm that, based on the matching occurrences of predicates of an XPE, determines whether the expression is matched. We then explore two optimized organizations of XPE predicates to more efficiently identify matching candidates.

\subsubsection{Determining a Matching Expression}

This section describes how to determine a matching XPE from the matching occurrence information of its constituent predicates. An XPE, $s$, is the ordered set of predicates, $s=$ pre $_{1} \mapsto$ pre $_{2} \mapsto \ldots \mapsto$ pre $_{n}$. The predicate matching result for the predicate over one tag and two tags is the set of occurrence numbers and occurrence number pairs, respectively. In our notation (and implementation) we omit the tag names and only keep track of occurrence numbers, as tag information is no longer needed. For example, in Table 1 , the predicate matching occurrence results for expression, $a / / b / c$ are $\{(1,1),(1,2),(2,2)\} \mapsto\{(1,1),(2,2)\}$. Here, the first tuple $(1,1)$ specifies that the first predicate of $a / / b / c$ is matched by the first tag, $a$, and the first tag, $b$, in the document path. The second tuple $(1,1)$ specifies that the second predicate of $a / / b / c$ is matched by the first $\operatorname{tag}, b$, and the first tag, $c$, in the XML document path. Not each combination of matching occurrence results of predicates are a true match (see Example 2). The combination is a true match if and only if the second occurrence number of pre $_{i-1}$ is the same as the first occurrence number of pre $_{i}(2 \leq i \leq n)$. A correct combination has the following format: $\left(o_{1}^{1}, o_{2}^{1}\right),\left(o_{1}^{2}, o_{2}^{2}\right), \ldots,\left(o_{1}^{n}, o_{2}^{n}\right)$, where $\left(o_{1}^{i}, o_{2}^{i}\right)$ is

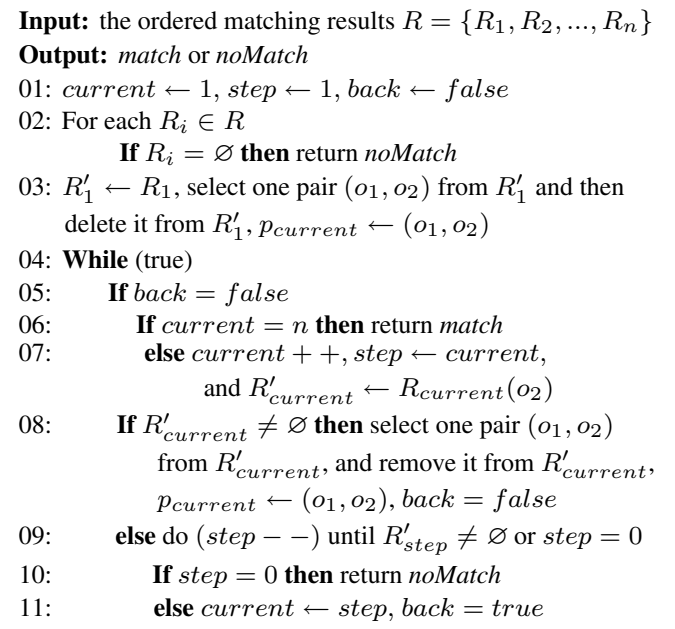

Figure 2. Occurrence Determination Algo.

selected from the predicate matching results of pre $_{i}$, and $o_{2}^{i-1}=o_{1}^{i}$ holds for each $i(2 \leq i \leq n)$.

Depending on the predicate type, a matching occurrence result has one or two values, i.e., one per tag name variable in the predicate. In our notation, to simplify the discussion, we duplicate the occurrence result, if only one value is present. For relative predicates, two different values are required and cannot be avoided (i.e., one per matching tag name variable in the document path.)

The problem of finding a sequence of occurrence numbers is a constraint satisfaction problem [6], where the constraints are $o_{2}^{i-1}=o_{1}^{i}$ for each $i(2 \leq i \leq n)$. We use a backtracking algorithm [6,3] to solve this constraint satisfaction problem. Our algorithm is shown in Figure 2.

The input to the algorithm are predicate matching results, $R=\left\{R_{1}, R_{2}, \ldots, R_{n}\right\}$, for the given XPE, in correct order, where $R_{i}$ is the predicate matching result for pre $_{i}$. The algorithm exits with match if it finds a correct match, and returns noMatch if it finds no matching combination. The algorithm works by traversing the space of partial matching solutions (i.e., sequences of matching occurrence numbers) in a depth-first manner and prunes the search space by ruling out discontinuing occurrences (i.e., occurrences such as $(1,1)$ and $(2,3)$, for instance, which do not constitute matching candidates.) The algorithm stops as a matching sequence is found. Were we to continue the algorithm, all possible matches would be found. To implement the specified matching semantic we only need to determine one match, not all possible matches.

We now discuss the algorithm in detail. The variables, initialized in line 1 are current representing the current predicate, step representing the backtracking depth of the algorithm, and back a flag to record the algorithm's "direction" - forward or backward. The algorithm immediately 
returns noMatch if there is any predicate without matching result among the input (line 2). Next, the algorithm (randomly) selects one pair, $\left(o_{1}, o_{2}\right)$, from $R_{1}^{\prime}$, removes it, and lets $R_{1}^{\prime}$ be the set of available pairs for pre $_{1}$ (line 3 ). Then the algorithm selects all pairs, whose first occurrence number is $o_{2}$, from $R_{2}$, and stores these pairs in $R_{2}^{\prime}$. If $R_{2}^{\prime}$ is $\varnothing$, then this indicates that no correct combination could start with $\left(o_{1}, o_{2}\right)$, therefore, we have to select another occurrence number pair from $R_{1}^{\prime}$. This step continues for all $i$. That is, select one pair, $\left(o_{1}^{i}, o_{2}^{i}\right)$, from $R_{i}^{\prime}$, which is the current selected pair for pre $_{i}$, and removes it from $R_{i}^{\prime}$ (line 8). We build $R_{i+1}^{\prime}$ by selecting pairs, whose first occurrence number is $o_{2}^{i}$, from $R_{i+1}$. $R_{i+1}^{\prime}$ is the set of available matching results for pre $_{i+1}$ (line 7). If $R_{i+1}^{\prime}=\varnothing$, then there is no correct choice for currently selected occurrence numbers, $\left(o_{1}^{i}, o_{2}^{i}\right)$. We, therefore, backtrack to $i$, chose the next available, $\left(o_{1}^{i}, o_{2}^{i}\right)$, if $R_{i}^{\prime} \neq \varnothing$. The algorithm returns match as the first $\left(o_{1}^{n}, o_{2}^{n}\right)$ (line 6) is found, and returns noMatch, if $R_{1}^{\prime}$ is $\varnothing$ (line 10).

\subsubsection{Optimized Expression Organizations}

The objective is to apply the occurrence determination algorithm as few times as possible. The algorithm does not have to be applied, if for a given XPE, one or more of its predicates is not satisfied. The algorithm does not have to be applied either, if we have established that an expression subsuming other expressions is satisfied, since the subsumed expressions will be satisfied as well. In this section, we exploit these observations to more advantageously organize the XPE predicates managed by our algorithm.

For two XPEs $s_{1}$ and $s_{2}$, we say that $s_{1}$ covers $s_{2}$ if and only if all publications that match $s_{2}$ also match $s_{1}$. As a result, if we know $s_{2}$ is matched by a publication, then we can draw the conclusion that $s_{1}$ is also matched by this publication without further evaluating it. That is, $s_{1}$ is subsumed by $s_{2}$. We find that XPEs, where one constitutes a prefix of the other one, are in a covering relation. Suppose that for two XPEs, $s_{1}:$ pre $_{1} \mapsto$ pre $_{2} \mapsto, \ldots, \mapsto$ pre $_{m}$ and $s_{2}:$ pre $_{1} \mapsto$ pre $_{2} \mapsto, \ldots, \mapsto$ pre $_{n}(1 \leq m \leq n)$, then, $s_{1}$ is the prefix of $s_{2}$. If one publication matches $s_{2}$, the occurrence determination algorithm will satisfy it, which indicates that there exists at least one correct combination, $\left(o_{1}^{1}, o_{2}^{1}\right),\left(o_{1}^{2}, o_{2}^{2}\right), \ldots,\left(o_{1}^{n}, o_{2}^{n}\right)$. As a result, the occurrence determination algorithm for $s_{1}$ will return a match as result as well, since there exists at least the combination, $\left(o_{1}^{1}, o_{2}^{1}\right),\left(o_{1}^{2}, o_{2}^{2}\right), \ldots,\left(o_{1}^{m}, o_{2}^{m}\right)$. That is, this publication also matches $s_{1}$. Therefore, we do not need to evaluate $s_{1}$, since $s_{1}$ covers $s_{2}$. Note, the covering relation also holds, if for two expressions, one constitutes a suffix or a contained expression of the other one. We exploit prefix-covering relation in this paper and postpone others to future work.

We use an XPE data structure that takes advantage of this prefix-covering relation among expressions. A fragment of our data structure representing expressions starting with

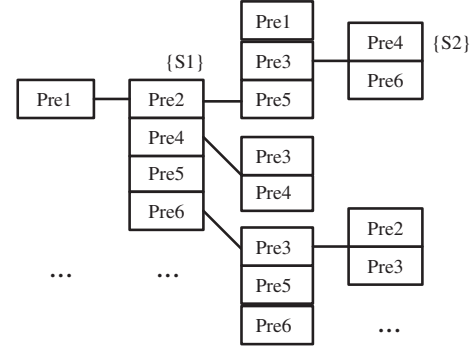

Figure 3. Fragment of XPE Index

pre $_{1}$ is shown in Figure 3. Expressions are indexed by their predicates. In the figure, we designate entire XPEs through marking the final predicate with XPE identifiers (sids). For example, $s_{2}:$ pre $_{1} \mapsto$ pre $_{2} \mapsto$ pre $_{3} \mapsto$ pre $_{4}$ represents an XPE. $s_{1}$ is a prefix of $s_{2}$, which indicates that $s_{2}$ covers $s_{1}$. Thus, we do not need to evaluate $s_{1}$ given a publication that satisfies $s_{2}$. The strategy in processing expressions in the final stage of our algorithm is to evaluate expressions that cover the most number of expressions first. In our current implementation we approximate this through selecting the longest expression first, deferring more refined heuristics to future work.

To reduce the number of times the occurrence determination algorithm is run, we consider a further optimized XPE layout. This is based on the observation that for a set of expressions that share common predicates, if the common predicates are not satisfied, all expressions do not need to be evaluated, since all of them will be false anyway. We refer to these common predicates as access predicates. In our approach, we cluster all expressions by their first predicate. More refined clustering schemes are imaginable. Also, better ways of determining candidate access predicates to cluster on come to mind. However, our primary objective is to determine whether this approach can yield better results to start with. The occurrence determination only runs, if the access predicates are satisfied, otherwise, the entire cluster is ruled out. The idea of an access predicate to rule out non-matching XPath expression sub-trees in our expression index has first been proposed by Fabret et al. [8] in the context of matching in publish/subscribe. However, that work was not about filtering XML documents through large volumes of XPEs. Also, the access predicate was applied to subscriptions not XPE expression trees.

\section{Evaluation}

\subsection{Experimental Setup}

In this section, we experimentally evaluate the performance of our algorithms based on the above described encoding. We also evaluate the two ways of organizing XPath expression predicates - prefix covering and access predi- 
cate. We compare our algorithms with two popular XML filtering techniques - YFilter [7] and Index-Filter [4]. We chose YFilter, as it has been shown to have superior performance over alternative approaches [7]. Yfilter is also representative for the class of automaton-based approaches. We chose Index-Filter, as one representative example of algorithms from the class of index-based filtering techniques. All algorithms are implemented in C. We modify the IndexFilter algorithm to stop after determining one match. The original algorithm is applied to find all matches in the XML document for each XPE. We perform all experiments on a computer with an Intel Xeon $2.4 \mathrm{GHz}$ processor with $2 \mathrm{~GB}$ RAM running Linux Redhat 8.

For generating the XPE workload, we used the XPath generator released by Diao et al. [7]. For generating the XML document workload, we used the IBM XML Generator [1]. This generator was used with default parameters except that we varied the maximum number of levels of the resulting XML documents from 6 to 10. This was set consistently with the maximum length of XPEs. In order to generate these workloads, we used two different DTDs: the NITF (News Industry Text Format) DTD ${ }^{4}$ and the PSD (Protein Sequence Database) DTD ${ }^{5}$. For each DTD, we generated 500 XML documents. The average size of our XML document is around $8.77 \mathrm{~KB}$ containing on average 140 tags. For each algorithm, we filtered all 500 XML documents against the corresponding XPath workload. All reported results are averaged over this set. All experiments report the total filtering time as the main performance metric. This time includes the time of parsing the XML document (for our algorithms this also includes the time to generate the encodings), matching the document against the XPE workload and collecting all results. As we show later, parsing time is negligible, unlike the results reported by other researchers. In our experiments, we suppose that all XPEs are processed before any XML documents are matched. That is, the time to process XPEs is not included in the filter time. XPath insertion time is an interesting metric, but not considered here, due to space limitations. Note, in our approach, all insertion operations are constant time and the number of predicates encoding an XPE is linear in the number of location steps in an expression.

\subsection{Varying the Number of XPEs}

We first evaluate the scalability of the algorithms by varying the number of XPEs. First, we look at distinct expressions and then at workloads that contain duplicate expressions.

Distinct Expression Workload: In this experiment, first, we compare three of our predicate-based filtering algorithm variants against each other to determine the best one under the given workloads. The basic matching algo-

\footnotetext{
${ }^{4}$ http://www.nitf.org

${ }^{5} \mathrm{http}: / /$ pir.georgetown.edu
}

rithm without any optimizations (basic), the matching algorithm with prefix covering (basic-pc) and the matching algorithm with prefix covering and access predicate (basicpc-ap). We generate two XPE workloads for NITF and PSD, respectively, and set flag of distinct vs. non-distinct $(D)$ to true, maximum length of XPE $(L)$ to 6 , probability of " $*$ " occurring at a location step $(W)$ and probability of "//" occurring at a location step $(D O)$ to 0.2 , respectively.

The number of distinct expressions ranges from 25,000 to 125,000 and from 1,000 to 10,000 for NITF and PSD, respectively. The results for NITF are shown in Figure 4(a). As can be seen, each algorithm scales linearly with the number of distinct expressions processed. The algorithm basic performs worst. The algorithm basic-pc performs consistently better. The improvement is a direct consequence of the prefix covering technique.

The prefix covering technique is sensitive to the number of covering relations in the expressions. It is also sensitive to the overall percentage of matched expressions. The higher the percentage of matched expressions, the fewer times the occurrence determination algorithm is expected to execute. In the NITF workload, the generated expressions are extremely selective: the percentage of matched expressions is on average $6 \%$. Therefore, the prefix covering technique can only contribute little, even if there is a large number of covering relations among expressions. If, in addition, we apply the access predicate technique in the basic-pc-ap version of the algorithm, we obtain the best results for this workload.

Figure 4(b) presents the results for the PSD workload. The overall trends observed above are similar for this workload. However, the main difference is the much higher percentage of match, which is $75 \%$ for this data set. Here, the prefix covering technique significantly contributes to reducing the processing time.

In Figure 4(a) and (b) we also compare our algorithms with the alternative approaches. We find that for a low percentage of matching in the workload (6\% in NITF in Figure 4(a)) YFilter performs better than our basic-pcap algorithm, if the number of expressions is greater than 100,000 . YFilter can take better advantage of the high selectivity of the NITF workload. If only a small number of expressions are matched, the execution of the NFA extends to only a very limited number of states as the XML tag elements do not trigger new transitions. In contrast, our algorithm translates all the XML paths into attribute-value pairs and matches them against the existing predicates, whether the paths match any predicates or not. The Index-Filter algorithm performs worst and takes almost twice as much time as YFilter. Our workload is not favorable for IndexFilter. Its strength is the processing of small number of expressions over large XML documents.

However, in a contrasting scenario, where the percentage 

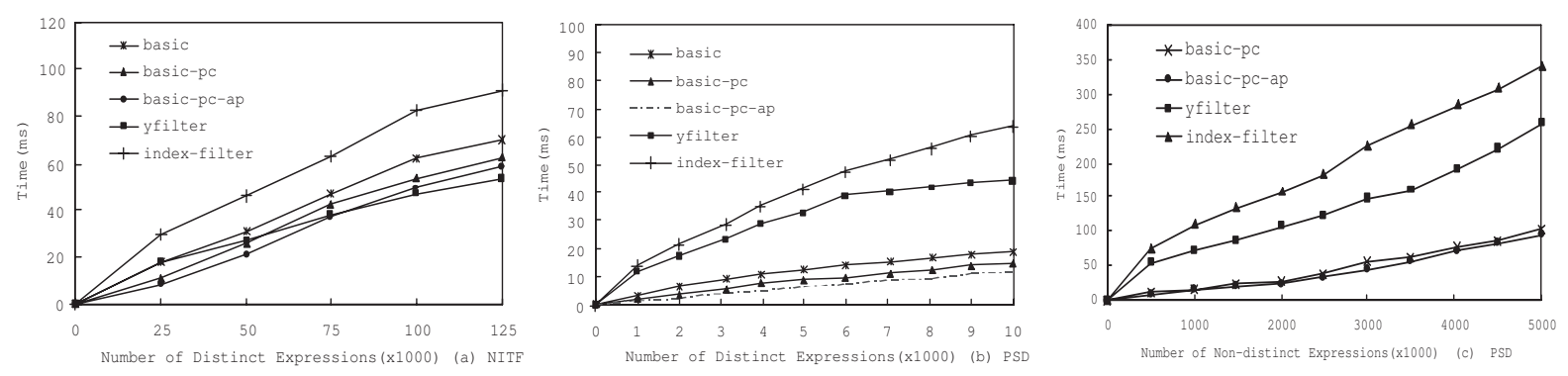

Figure 4. Varying the number of XPEs (Each data point reports an average over 500 documents)

of matching in the workload is high, as for the PSD DTD in Figure 4(b) with $75 \%$ matching, the difference between YFilter and our algorithms becomes much more significant. As compared to the above case, the situation is reversed with our algorithms performing significantly better. The YFilter NFA has to undergo a large number of transitions and touches a lot of states, whereas our algorithm amortizes the work done in predicate matching and XPE matching in determining the large number of matches. Index-Filter still performs least favorable, as explained above.

Duplicate Expression Workload: In large, Internetscale filtering system the expression workloads are likely to contain duplicate expressions. These duplicates represent the common and shared interests among users. In this experiment, we vary the number of expressions from half a million to 5 millions and set $D$ to false, all other parameters are the same as before. The number of distinct expressions ranges from 55,000 to 167,000 and from 5,500 to 10,000 for NITF and PSD DTDs, respectively. Figures 4(c) shows the results for PSD DTD. The NITF results are similar with distinct expression experiment (not included, due to space limitations). For both DTDs, all algorithms scale linearly when increasing the number of expressions. In the NITF workload, our algorithm performs slightly better than YFilter. basic-pc-ap and yfilter perform 121.5 and 137.3 ms over 5 millions of duplicate expressions, respectively, which indicates that our algorithm accommodates duplicate expressions in the workload slightly better. For the PSD DTD, our algorithm outperforms YFilter in the larger expression regimes by more than half of its matching time. Again, this is due to the high percentage of matching.

\subsection{Effect of Wildcards and Descendants}

In this section, we investigate the impact of the probability of wildcards and descendant operators on matching time. We only report the NITF workload, since the PSD workload has the similar results. For wildcards, we set $D O$ to 0.2 and vary $W$ from 0 to 0.9 ; for descendant operators, we set $W$ to 0.2 and vary $D O$ from 0 to 0.9 . Both workloads contain 2 million expressions. We show the NITF results for increasing wildcard in Figure 5. The results for varying descendant operator probability are similar and not

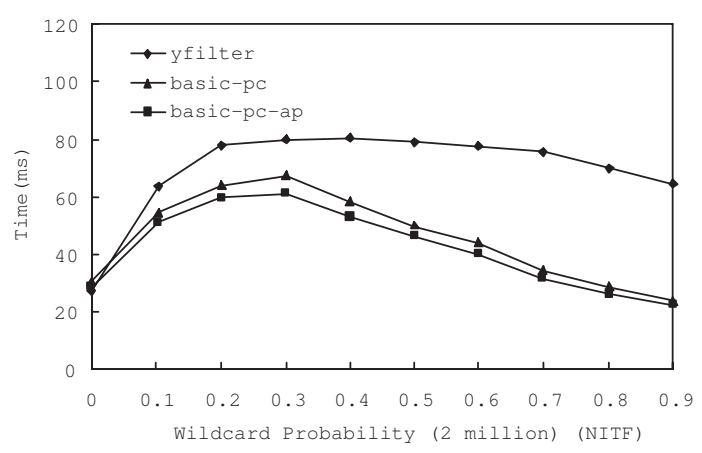

Figure 5. Varying the wildcard probability

included due to space limitations. With the increase in wildcard probability, the number of predicates first grows because the addition of wildcards will increase the number of predicates, adding new predicates with increasing range values to the existing predicates. As $W$ is further increased, the overall number of predicates begins to decrease resulting in fewer distinct predicates. The turning point here is 0.3 . After this point, the expressions become very similar to each other and more overlapping and duplicate expressions are in the workload resulting in less matching time. For descendent operator, with increasing probability, the number of predicates first increases because of the addition of further relative, greater-than-or-equal predicates and, finally, a decrease because expressions overlap and more duplicates come about in the workload.

In YFilter, the increase of $W$ and $D O$ will increase the non-determinism of the underlying NFA. Even when $W$ is set to 0.9 , at which point the number of distinct expressions has significantly decreased, YFilter's performance does not improve significantly like our algorithm. This happens because more states of the NFA are touched in filtering. The wildcards are matched by any XML element leading to many state transitions. A similar argument applies to varying $D O$.

Index-Filter is not evaluated for increasing $W$. The orig- 
inal paper does not discuss how to handle wildcards. In our implementation, we just simply let wildcards match any XML elements, which makes the size of index stream of each node in the prefix tree augment rapidly, especially when the probability of wildcards is high. For descendant operator, it is also less sensitive to the varying probability like YFilter.

\subsection{Matching Cost Breakdown}

In this experiment we aim to understand where the time is spent in our algorithm. The total filtering time in all our experiments includes the time spent on parsing the XML document, performing predicate matching and performing expression matching. In this experiment we isolate these times to understand the impact of the different stages of the algorithm. Our evaluations show that parsing time is negligible compared to the total filtering time. The average parsing time for NITF and PSD XML documents is only 314 and 355 microseconds, respectively. We, therefore, do not show these numbers, as they are too small. In the experiment, we used the same workload as in the experiment with duplicate expressions. Table 2 shows the cost breakdown for predicate matching, expression matching (i.e., occurrence determination), and other computations (i.e., collecting the results). We only show NITF workload results (PSD results are similar). As can be seen in the table, the cost for expression matching dominates. As the number of expression increases, the time spent on predicate matching rises less severely than the time spent on expression matching. As more expressions are processed, the number of distinct predicates does not increase to the same degree as the number of expressions. This is because our algorithm takes advantage of duplicated predicates storing and processing them only once. The numbers of distinct predicates that are managed in our algorithm for the shown data sets are 4019, 4593, 5150, 5521, and 5843, which changes comparatively little compared to the change in expression numbers (i.e., from $1000 \mathrm{~K}$ to $5000 \mathrm{~K}$ ).

Table 2. Matching Cost Breakdown

\begin{tabular}{|c|c|c|c|c|c|}
\hline Matching Cost & \multicolumn{5}{|c|}{ Number of Expressions (NITF) } \\
\cline { 2 - 6 } Breakdown (in ms) & $1000 \mathrm{k}$ & $2000 \mathrm{k}$ & $3000 \mathrm{k}$ & $4000 \mathrm{k}$ & $5000 \mathrm{k}$ \\
\hline Pred. Matching & 10.61 & 13.2 & 16.57 & 18.51 & 21.19 \\
\hline XPE Matching & 26.49 & 35.72 & 46.17 & 59.9 & 74.93 \\
\hline Other & 11.02 & 16.85 & 19.22 & 22.86 & 25.5 \\
\hline
\end{tabular}

\section{Conclusions}

In this paper, we have developed and evaluated a novel matching algorithm to solve the XML/XPath filtering problem for filtering XML documents against large numbers of XPEs. The novel ideas underlying our algorithm are to encode XPEs as Boolean predicates, encode XML documents as sets of (attribute, value)-pairs, and efficiently evaluate these tuples over the Boolean predicates. This constitutes an altogether new approach for solving the XML filtering problem, next to the popular automaton-based and indexbased filtering algorithms.

The strength of our approach is the great potential to take advantage of overlap in different XPEs. The overlap exploitation is manifest in the predicate encoding that stores and processes distinct predicates once. We have experimentally evaluated the performance of our algorithm and have constructively proven its scalability for processing of Internet-scale workloads (millions of XPEs and matching performance in the millisecond range). We have evaluated our approach next to common alternatives, namely YFilter and Index-Filter. This comparison gave rise to interesting trade-offs and novel insights.

For an XPE workload with a high percentage of matched expressions, our algorithm is more efficient than the alternative approaches investigated. For a contrasting workload with a very low matching percentage, YFilter outperformed our algorithm. For workloads that are based on expressions that are characterized by many wildcards and descendant operators, our algorithm consistently outperformed alternatives.

\section{References}

[1] D. A.L.Diaz. XML generator, Sept. 2003.

[2] M. Altinel and M. J. Franklin. Efficient filtering of XML documents for selective dissemination of information. $V L D B^{\prime} 02$.

[3] J. Bitner and E. M. Reingold. Backtracking programming techniques. CACM, 18(11):651-656, 1975.

[4] N. Bruno, L. Gravano, and N. Doudas. Navigation-vs. indexbased XML multi-query processing. In ICDE, 2003.

[5] C. Y. Chan, P. Felber, M. N. Garofalakis, and R. Rastogi. Efficient filtering of XML documents with XPath expressions. In ICDE, 2002.

[6] R. Dechter and D. Frost. Backjump-based backtracking algorithms for constraint satisfaction problems. Artificial Intelligence, 136(2):147-188, 2002.

[7] Y. Diao, M. Altinel, M. J. Franklin, H. Zhang, and P. Fischer. Path sharing and predicate evaluation for high-performance XML filtering. ACM TDBS, 28(4):467-516, 2003.

[8] F. Fabret, H.-A. Jacobsen, F. Llirbat, J. Pereira, K. Ross, and D. Shasha. Filtering algorithms and implementation for very fast publish/subscribe systems. In SIGMOD, 2001.

[9] T. J. Green, G. Miklau, M. Onizuka, and D. Suciu. Processing XML streams with deterministic automata. In ICDT, 2002.

[10] A. K. Gupta and D. Suciu. Stream processing of xpath queries with predicates. In SIGMOD, 2003.

[11] S. Hou and H.-A. Jacobsen. Predicate-based filtering of xpath expressions. Technical Report CSRG-514 at ftp://www.cs.toronto.edu/pub/reports/csrg/514, Department of Computer Science, University of Toronto, 2005.

[12] L. V. S. Lakshmanan and S. Parthasarathy. On efficient matching of streaming XML documents and queries. In $E x$ tending Database Technology, 2002.

[13] F. Tian, B. Reinwald, H. Pirahesh, T. Mayr, and J. Mylly maki. Implementing a scalable XML publish/subscribe system using relational database systems. In SIGMOD, 2004. 